\title{
The $p$-Version of the Finite Element Method for Constraint Boundary Conditions
}

\author{
By I. Babuska*and Manil Suri**
}

\begin{abstract}
The paper addresses the implementation of general constraint boundary conditions for a system of equations by the $p$-version of the finite element method. By constraint boundary conditions we mean conditions where some relation between the components is prescribed at the boundary. Optimal error bounds are proven.
\end{abstract}

1. Introduction. There is a large variety of boundary conditions for systems of differential equations of elliptic type. Some physically natural conditions may be formulated by a variational approach through constraint conditions. For example, the two-dimensional elasticity problem can be formulated as the minimization of a quadratic functional $F(u), u=\left(u_{1}, u_{2}\right)$, over a set $H$ satisfying

$$
\left(H_{0}^{1}(\Omega)\right)^{2} \subset H \subset\left(H^{1}(\Omega)\right)^{2} .
$$

Selections of $H$ then characterize the boundary conditions.

Obviously, the choice $H=\left(H_{0}^{1}(\Omega)\right)^{2}$ induces the (essential) Dirichlet conditions, i.e., the displacement is given on $\partial \Omega$, while $H=\left(H^{1}(\Omega)\right)^{2}$ induces the (natural) Neumann conditions, i.e., the tractions are prescribed on $\partial \Omega$. In addition to these classical conditions, other types are important in applications. One of these conditions is characterized by

$$
H=\left\{\left(u_{1}, u_{2}\right) \in\left(H^{1}(\Omega)\right)^{2} \mid u_{1} \varphi_{1}(s)+u_{2} \varphi_{2}(s)=0 \text { on } \partial \Omega\right\}
$$

where $\varphi_{1}$ and $\varphi_{2}$ are given functions defined on $\partial \Omega$. These conditions are in the most simple case the symmetry conditions and in general, traction-free constraints at the boundary.

So far we have only mentioned homogeneous boundary conditions. Nonhomogeneous conditions are defined in the usual way, when the minimization of $F$ is over a hyperplane $H_{v}=\left\{u+v \mid u \in H, v \in\left(H^{1}(\Omega)\right)^{2}\right\}$.

The constraint boundary condition we mentioned above is a type of essential condition. Hence, when solving such problems by the finite element method in general, and by the $p$ or $h$ - $p$ versions in particular, we face the problem of implementing the nonhomogeneous boundary conditions (which are outside the finite element space).

Received May 13, 1987.

1980 Mathematics Subject Classification (1985 Revision). Primary 65N30, 41A10, 41A25.

*Partially supported by ONR Contract N00014-85-K-0169.

** Research partially supported by the Air Force Office of Scientific Research, Air Force Systems Command, USAF, under Grant No. AFOSR-85-0322. 
The $p$ and $h$ - $p$ versions are recent developments, where $p$, the degree of the elements used, is not fixed but is increasing. This is in contrast to the classical $h$ version, where the degree $p$ is kept fixed. The first commercial programs available are PROBE (Noetic Tech., St. Louis) and FIESTA (ISMES, Bergamo, Italy).

The implementation of Dirichlet boundary conditions for the $p$-version of the finite element method has been addressed by us in [2] and [4]. A general survey on the state of the art of the $p$ and $h-p$ versions may be found in [1].

In this paper we will address the implementation of the constraint conditions (1.1) in a simplified setting (to avoid notational difficulties). Section 2 deals with preliminaries and notation. In Section 3 we formulate an abstract approach, and based on it prove that the suggested finite element formulation of the constraint boundary condition leads to the optimal rate of convergence of the $p$-version. Section 4 addresses some implementational aspects.

\section{Basic Notation and Preliminaries.}

2.1. The Sobolev Spaces. Let $\mathbf{R}^{2}$ be the two-dimensional Euclidean space, $x=$ $\left(x_{1}, x_{2}\right) \in \mathbf{R}^{2}$. Let $\Omega \subset \mathbf{R}^{2}$ be a bounded Lipschitzian domain with the boundary $\Gamma=\partial \Omega$. We will assume that $\Gamma$ is a Jordan curve, $\Gamma=\bigcup_{i=1}^{m} \bar{\Gamma}_{i}$, where $\Gamma_{i}$ are smooth open arcs with parametric description

$$
\Gamma_{i}=\left\{\left(x_{1}, x_{2}\right)\left|x_{1}=x_{i, 1}(\xi), x_{2}=x_{i, 2}(\xi),\right| \xi \mid<1\right\}, \quad i=1, \ldots, m .
$$

Denoting $I=(-1,1), \Gamma_{i}$ is obviously the image of $I$ by the mapping

$$
F_{i}=\left\{x_{i, 1}, x_{i, 2}\right\},
$$

i.e., $\Gamma_{i}=F_{i}(I)$. If $u(s)$ is defined on $\Gamma_{i}$, then by $U(\xi)=u\left(F_{i}(\xi)\right)$ we denote its transform on $I$. The ends of $\Gamma_{i}$ will be called vertices and denoted by $A_{i}=$ $\left(x_{i, 1}(-1), x_{i, 2}(-1)\right), B_{i}=\left(x_{i, 1}(1), x_{i, 2}(1)\right)$. We will further assume that $B_{i}=A_{i+1}$, $B_{m}=A_{1}, i=1, \ldots, m$. By this, the orientation of $\Gamma_{i}$ is established. In general we will denote the vertices by $A_{i}\left(=B_{i-1}\right), i=1, \ldots, m$. The scheme of the domain and the pertinent notation is shown in Figure 2.1.

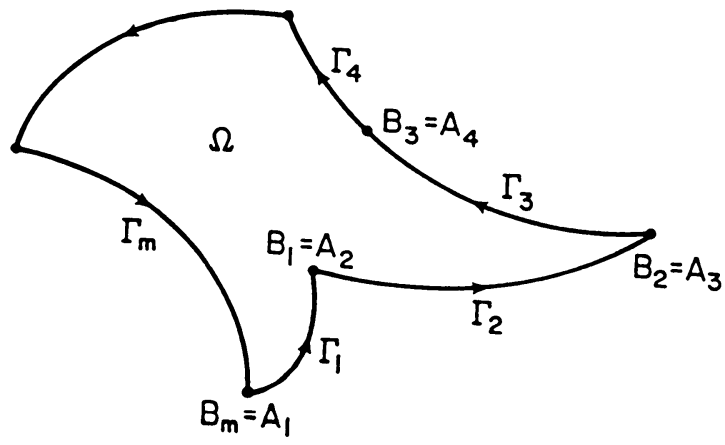

FIGURE 2.1

Scheme of the domain and notation.

Remark 2.1. We assumed that the domain $\Omega$ is simply connected. This assumption has been made only for notational simplicity.

Remark 2.2. We assumed that the domain is Lipschitzian. Once more, our results are valid (with proper modification) in the case when, for example, some arcs coincide (as in the case of the slit domain). 
Remark 2.3. We have assumed that the $\operatorname{arcs} \Gamma_{i}$ are sufficiently smooth. For the sake of simplicity we assume that they are $C^{\infty}$ arcs (i.e., the functions $x_{i, j}$, $i=1, \ldots, m, j=1,2$, are $C^{\infty}$ functions).

By $H^{k}(\Omega), k \geq 0$ integer, we denote the usual Sobolev space of functions with square integrable derivatives on $\Omega$. The norm will be denoted by $\|\cdot\|_{H^{k}(\Omega)}$. If $l<q<l+1, l \geq 0$ integer, then we define $H^{q}(\Omega)=\left(H^{l}(\Omega), H^{l+1}(\Omega)\right)_{\theta}, \theta=q-l$, where by $(\cdot, \cdot)_{\theta}$ we denote the usual interpolated space using the $K$-method (see $[5])$. The scalar product $(\cdot, \cdot)_{H^{q}}$ and the norm $\|\cdot\|_{H^{q}(\Omega)}$ are defined accordingly.

By $C^{k}(\bar{\Omega}), k \geq 0$ integer, we denote the space of all functions with $k$ continuous derivatives on $\bar{\Omega}$. It is possible to show that $H^{k}(\Omega) \hookrightarrow C^{0}(\bar{\Omega})$ for $k>1$, where by $\hookrightarrow$ we denote continuous imbedding. On the other hand, $H^{1}(\Omega) \not \subset C^{0}(\bar{\Omega})$.

For $I=(-1,1), H^{k}(I), k \geq 0$ is defined analogously as before. If $k>1 / 2$, then $H^{k}(I) \hookrightarrow C^{0}(\bar{I})$, but $H^{k}(I) \not \subset C^{0}(\bar{I})$ for $k \leq 1 / 2$.

So far we have defined $H^{k}(I), k \geq 0$. We will also be interested in $H^{k}(I), k<0$. We define for $k \geq 0$

$$
\|u\|_{H^{-k}(I)}=\sup _{\substack{v \neq 0 \\ v \in H^{k}(I)}} \frac{\int_{-1}^{1} u v d x}{\|v\|_{H^{k}(I)}} .
$$

(Let us remark that sometimes (see, e.g., [5]) our space $H^{-k}(I)$ is denoted by $\left(H^{k}(I)\right)^{\prime}$, whereas $H^{-k}(I)$ is used to denote the dual space of $H_{0}^{k}(I)$.)

If $u$ is defined on $\Gamma_{i}$, then we define

$$
\begin{gathered}
H^{k}\left(\Gamma_{i}\right)=\left\{u \mid u\left(F_{i}(\xi)\right)=U(\xi) \in H^{k}(I)\right\}, \\
\|u\|_{H^{q}\left(\Gamma_{i}\right)}=\|U\|_{H^{k}(I)} .
\end{gathered}
$$

So far we have considered only scalar functions on $\Omega$ and $I$. The spaces of vector functions are defined by Cartesian products, ${ }^{2} H^{k}(\Omega)=\left(H^{k}(\Omega)\right)^{2}$.

Now let

$$
\begin{aligned}
& Q=\left\{\left(x_{1}, x_{2}\right)|| x_{1}|<1,| x_{2} \mid<1\right\}, \\
& \gamma_{1}^{Q}=\left\{\left(x_{1}, x_{2}\right)|| x_{1} \mid<1, x_{2}=-1\right\} .
\end{aligned}
$$

$Q$ will be called the standard square and $\gamma_{i}^{Q}, i=1,2,3,4$, its sides $\left(\gamma_{i}^{Q}, i=2,3,4\right.$, are defined analogously to $\gamma_{1}^{Q}$ in an obvious way). Let

$$
\begin{gathered}
T=\left\{\left(x_{1}, x_{2}\right)|| x_{1} \mid<1,0<x_{2}<\left(1+x_{1}\right) \sqrt{3} \text { for } x_{1}<0,\right. \\
\left.0<x_{2}<\left(1-x_{1}\right) \sqrt{3} \text { for } x_{1}>0\right\}, \\
\gamma_{1}^{T}=\left\{\left(x_{1}, x_{2}\right)|| x_{1} \mid<1, x_{2}=0\right\} .
\end{gathered}
$$

$T$ will be called the standard triangle and $\gamma_{i}^{T}, i=1,2,3$, its sides.

Let us remark that the sides of $T$ and $Q$ are each of length 2. Later we will often not distinguish between $\gamma_{i}$ and $I$.

We now define

$$
\begin{gathered}
\mathscr{P}_{p}^{2}(Q)=\{u \mid u \text { is a polynomial of degree } \leq p \\
\text { in each variable } \left.x_{1} \text { and } x_{2} \text { over } Q\right\}, \\
\mathscr{P}_{p}^{1}(T)=\{u \mid u \text { is a polynomial of (total) degree } \leq p \text { on } T\}, \\
\mathscr{P}_{p}(I)=\{u \mid u \text { is a polynomial of degree } \leq p \text { on } I\} .
\end{gathered}
$$




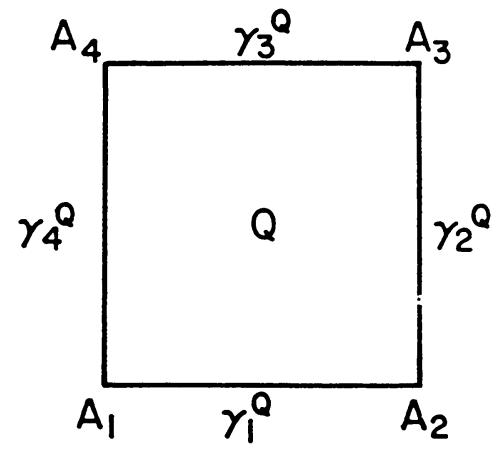

FIGURE 2.2

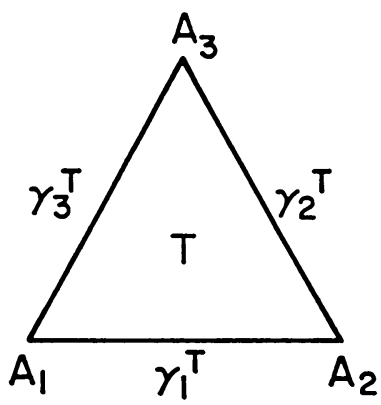

The scheme of the standard square and standard triangle.

We then have

LEMMA 2.1. Let $v \in H_{0}^{1}\left(\gamma_{1}^{Q}\right) \cap \mathscr{P}_{p}\left(\gamma_{1}^{q}\right)$ (respectively $\left.v \in H_{0}^{1}\left(\gamma_{1}^{T}\right) \cap \mathscr{P}_{p}\left(\gamma_{1}^{T}\right)\right)$ such that

respectively

$$
\|v\|_{H^{t}\left(\gamma_{1}^{Q}\right)} \leq p^{-(1-t)} A, \quad t=0,1
$$

$$
\|v\|_{H^{t}\left(\gamma_{1}^{T}\right)} \leq p^{-(1-t)} A, \quad t=0,1 .
$$

Then there exists $u \in \mathscr{P}_{p}^{2}(Q)$ (respectively $\mathscr{P}_{p}^{1}(T)$ ) such that $\left.u\right|_{\gamma_{1}^{Q}}=v$ (respectively $\left.\left.u\right|_{\gamma_{1}^{T}}=v\right),\left.u\right|_{\partial Q-\gamma_{1}^{Q}}=0$ (respectively $\left.\left.u\right|_{\partial T-\gamma_{1}^{T}}=0\right)$ and

$$
\|u\|_{H^{1}(Q)} \leq C p^{-1 / 2} A
$$

respectively

$$
\|u\|_{H^{1}(T)} \leq C p^{-1 / 2} A .
$$

For the proof see [2] or [3].

2.2. The Model Problem. Let

$$
{ }^{2} H_{0}^{1}(\Omega) \subset \mathscr{H}(\Omega) \subset{ }^{2} H^{1}(\Omega),
$$

where $\mathscr{H}(\Omega) \notin{ }^{2} H^{1}(\Omega)$ is closed in ${ }^{2} H^{1}(\Omega)$. $\mathscr{H}(\Omega)$ will be called the constraint space. Assume that there is given a continuous bilinear form $B(u, v)$ on ${ }^{2} H^{1}(\Omega) \times$ ${ }^{2} H^{1}(\Omega), u=\left(u_{1}, u_{2}\right), v=\left(v_{1}, v_{2}\right)$, such that

$$
B(u, u) \geq \gamma\|u\|_{2 H^{1}(\Omega)}^{2}, \gamma>0 \text { for any } u \in{ }^{2} H^{1}(\Omega) .
$$

Then, obviously, for any $G_{1}\left({ }^{2} H^{1}(\Omega)\right)^{\prime}$ there is a unique $u_{0} \in \mathscr{H}(\Omega)$ such that

$$
B\left(u_{0}, v\right)=G_{1}(v)
$$

holds for any $v \in \mathscr{H}(\Omega)$. We also have

$$
\left\|u_{0}\right\|^{2} H^{1}(\Omega) \leq C\left\|G_{1}\right\|_{\left({ }^{2} H^{1}(\Omega)\right)^{\prime}} .
$$

Denote $\mathscr{H}_{\rho}(\Omega)=\left\{u \in{ }^{2} H^{1}(\Omega), u-\rho \in \mathscr{H}(\Omega)\right\} . \mathscr{H}_{\rho}(\Omega)$ will be called the $\rho$-hyperplane. Then our model problem is given by: Find $u_{0} \in \mathscr{H}_{\rho}(\Omega)$ such that

$$
B\left(u_{0}, v\right)=G_{1}(v) \quad \forall v \in \mathscr{H}(\Omega) .
$$

We then have

$$
\left\|u_{0}\right\|_{{ }^{2} H^{1}(\Omega)} \leq C\left[\|\rho\|_{2 H^{1}(\Omega)}+\left\|G_{1}\right\|_{\left({ }^{2} H^{1}(\Omega)\right)^{\prime}}\right] .
$$


If $\rho=0$, then we will speak about a homogeneous constraint problem, while for $\rho \neq 0$ we will speak about a nonhomogeneous constraint problem. We call these constraint problems because $\mathscr{H}(\Omega) \neq{ }^{2} H^{1}(\Omega)$.

There are many constraint problems in applications. We will consider the one when

$$
\mathscr{H}(\Omega)=\left\{\left(u_{1}, u_{2}\right) \in{ }^{2} H^{1}(\Omega)\left|\sum_{l=1}^{2} \alpha_{k, l}^{(j)} u_{l}\right|_{\Gamma_{j}}=0, k=1,2, j=1, \ldots, m\right\},
$$

where $\alpha^{j}=\left\{\alpha_{k, l}^{(j)}\right\}$ are matrices of smooth functions on $\bar{\Gamma}_{j}$ (say $C^{\infty}\left(\bar{\Gamma}_{j}\right)$ ). Additional assumptions on $\left\{\alpha_{k, l}^{(j)}\right\}$ will be imposed later.

Obviously, when $\alpha_{k, k}=1, \alpha_{k, l}=0$ for $k \neq l$, we get Dirichlet boundary conditions (in general we get Dirichlet conditions when $\alpha^{j}$ have rank 2 for all $x \in \Gamma_{j}$ ). If $\left\{\alpha_{k, l}^{(j)}\right\}=0$, there is no constraint and we have the Neumann problem.

If $\alpha^{j}$ has rank 1 , then we can write the constraint on $\Gamma_{j}$ as

$$
\alpha_{1,1}^{(j)} u_{1}+\alpha_{1,2}^{(j)} u_{2}=0
$$

which will be written in the form

$$
\alpha^{(j)} u_{1}+\beta^{(j)} u_{2}=0 .
$$

Obviously, if $\rho=\left(\rho_{1}, \rho_{2}\right)$, then the nonhomogeneous constraint problem is characterized on $\Gamma_{j}$ by

$$
\alpha^{(j)} u_{1}+\beta^{(j)} u_{2}=\alpha^{(j)} \rho_{1}+\beta^{(j)} \rho_{2} .
$$

Problems of this type are common, for example in the theory of elasticity. For simplicity of the exposition and notation we will restrict ourselves to the model problem where

$$
B(u, v)=\int_{Q}\left(\frac{\partial u_{1}}{\partial x_{1}} \frac{\partial v_{1}}{\partial x_{1}}+\frac{\partial u_{1}}{\partial x_{1}} \frac{\partial v_{1}}{\partial x_{2}}+\frac{\partial u_{2}}{\partial x_{2}} \frac{\partial v_{2}}{\partial x_{1}}+\frac{\partial u_{2}}{\partial x_{2}} \frac{\partial v_{2}}{\partial x_{2}}\right.
$$

$$
\left.+u_{1} v_{1}+u_{2} v_{2}\right) d x_{1} d x_{2}
$$

and

$$
G_{1}(v)=\int_{\Omega}\left(f_{1} v_{1}+f_{2} v_{2}\right) d x_{1} d x_{2}, \quad f=\left(f_{1}, f_{2}\right) \in{ }^{2} H^{0}(\Omega) .
$$

Although we restrict ourselves to this special case, our results hold in general, e.g., for elasticity problems, etc.

We will assume that $\alpha_{k, l}^{(j)} \in C^{\infty}\left(\bar{\Gamma}_{j}\right)$. In practice, we have the nonhomogeneous constraint problem defined so that

(i) If $\left\{\alpha_{k, l}^{(j)}\right\}$ has rank 2 on $\bar{\Gamma}_{j}$ then the constraint is

$$
\sum_{l=1}^{2} \alpha_{k, l}^{(j)} u_{l}=g_{k}^{(j)}, \quad k=1,2
$$

where $\left(g_{1}^{(j)}, g_{2}^{(j)}\right)$ are defined on $\bar{\Gamma}_{j}$. Hence, we can obviously transform the above constraint equation to

$$
u_{1}=\tilde{g}_{1}^{(j)}, \quad u_{2}=\tilde{g}_{2}^{(j)}
$$


Because $\alpha_{k, l}^{(j)}$ are assumed to be smooth, we see that $\left\{\tilde{g}_{i}^{(j)}\right\}, i=1,2$, have the same smoothness as $\left\{g_{i}^{(j)}\right\}$.

(ii) If $\left\{\alpha_{k, l}^{(j)}\right\}$ has rank 1 , then the constraint equation is

$$
\alpha^{(j)} u_{1}+\beta^{(j)} u_{2}=g^{(j)}
$$

We add the condition

$$
\alpha^{(j)}+\beta^{(j)} \neq 0 \quad \text { on } \bar{\Gamma}_{j} .
$$

This enables us to transform the constraint equation to

$$
\alpha^{(j)} u_{1}+\beta^{(j)} u_{2}=\tilde{g}^{(j)} \quad \text { with } \alpha^{(j)}+\beta^{(j)}=1
$$

As formulated above, $\tilde{g}^{(j)}$ are defined separately on each $\bar{\Gamma}_{j}$. We will assume that $\tilde{g}^{(j)}$ satisfy consistency conditions, namely that there exists $\rho=\left(\rho_{1}, \rho_{2}\right) \in{ }^{2} H^{1}(\Omega)$ such that

$$
\tilde{g}_{1}^{(j)}=\left.\rho_{1}\right|_{\bar{\Gamma}_{j}}, \tilde{g}_{2}^{(j)}=\left.\rho_{2}\right|_{\bar{\Gamma}_{j}}
$$

respectively

$$
\left.\alpha^{(j)} \rho_{1}\right|_{\bar{\Gamma}_{j}}+\left.\beta^{(j)} \rho_{2}\right|_{\bar{\Gamma}_{j}}=\tilde{g}^{(j)} .
$$

These conditions have to be imposed especially at the vertices of $\Omega$.

The sides $\Gamma_{j}$ where the constraint (2.6) is imposed will be called total constraint sides, while $\Gamma_{j}$ where the constraint (2.7) is imposed will be called partial constraint sides. We will enumerate the total constraint sides as $\Gamma_{i_{j}}, j=1, \ldots, m_{1}$, and the partial constraint sides as $\Gamma_{i_{j}}, j=m_{1}+1, \ldots, m$.

2.3. The $p$-Version of the Finite Element Method. Assume that the domain $\Omega$ has been partitioned into a finite number of subdomains $\Omega_{i}$, i.e., $\bar{\Omega}=\bigcup_{i=1}^{n} \bar{\Omega}_{i}$. We shall assume that $\Omega_{i}$ is the curvilinear quadrilateral

$$
\Omega_{i}=\mathscr{F}_{i}(Q)
$$

or curvilinear triangle

$$
\Omega_{i}=\mathscr{F}_{i}(T),
$$

where $Q$ and $T$ are the standard square and triangle, respectively. The domains $\Omega_{i}$ will be called elements. We will assume that $\mathscr{F}_{i}^{-1}$ is a smooth one-to-one mapping of $\Omega_{i}$ onto $Q$, respectively $T$. It is obvious what the vertices and sides of $\Omega_{i}$ correspond to. If $\gamma$ is a side of $\Omega_{i}$, then $\mathscr{F}_{i}$ induces mapping $F_{i}$ of $I$ onto $\gamma$ (realizing that all the sides of the standard square and triangle have the same length as $I$ ).

We shall assume the following about the partition and the mappings $F_{i}$ :

$(\alpha)$ If $\bar{\Omega}_{i} \cap \bar{\Omega}_{j}=R_{i, j} \neq \varnothing$, then $R_{i, j}$ is either a common vertex or a side of both $\Omega_{i}$ and $\Omega_{j}$.

( $\beta$ ) If $R_{i, j}=\gamma_{i, j}$, then we will assume that the mappings $F$ of $I$ onto $\gamma_{i, j}$ induced by the mappings $\mathscr{F}_{i}$ and $\mathscr{F}_{j}$ are identical. We denote $F$ by $F_{i, j}$. This implies the following: Let $A, B$, the vertices of $\Omega_{i}$ and $\Omega_{j}$, be the end points of $\gamma_{i, j}$. Assume that $\left(a_{1}, b_{1}\right)$ and $\left(a_{2}, b_{2}\right)$ are the end points of the sides $\gamma_{k}^{Q}$ or $\gamma_{l}^{T}$ such that $\mathscr{F}_{i}\left(a_{1}\right)=\mathscr{F}_{j}\left(a_{2}\right)=A, \mathscr{F}_{i}\left(b_{1}\right)=\mathscr{F}_{j}\left(b_{2}\right)=B$. Then, if $C \in \gamma_{i, j}$ and $C=\mathscr{F}_{i}\left(c_{1}\right)=\mathscr{F}_{j}\left(c_{2}\right), \overline{a_{1} c_{1}}=\overline{a_{2} c_{2}}$ and $\overline{c_{1} b_{1}}=\overline{c_{2} b_{2}}$. 
Since we assumed that $\mathscr{F}_{i}$ are smooth mappings, the vertices of $\Omega$ necessarily have to coincide with some of the vertices of $\Omega_{i}$. We will further assume that for any $\Gamma_{j}$ there is an element $\Omega_{i}$ such that one of its sides coincides with $\Gamma_{j}$. This assumption is made without any loss of generality.

Denote now

$$
\begin{gathered}
1 \mathscr{P}_{p}(\Omega)=\left\{u \in H^{1}(\Omega)|u|_{\Omega_{i}}\left(\mathscr{F}_{i}(\xi)\right) \in \mathscr{P}_{p}^{2}(Q) \text { if } \Omega_{1}\right. \text { is a quadrilateral } \\
\text { and } \left.\left.u\right|_{\Omega_{i}}\left(\mathscr{F}_{i}(\xi)\right) \in \mathscr{P}_{p}^{1}(T) \text { if } \Omega_{i} \text { is a triangle }\right\}, \\
2 \mathscr{P}_{p}(\Omega)=\left({ }^{1} \mathscr{P}_{p}(\Omega)\right)^{2}, \\
2 \mathscr{P}_{p}(\gamma)=\left\{u\left|u_{j}\right|_{\gamma}\left(F_{i}(\xi)\right) \in \mathscr{P}_{p}(I), j=1,2\right\} .
\end{gathered}
$$

Here, $\gamma=\Gamma_{i}$ or any side of an element. Let us define the constraint space $\mathscr{H}^{\mathscr{P}}(\Omega) \subset$ ${ }^{2} \mathscr{P}_{p}(\Omega)$ as follows:

(i) If $\Gamma_{j}$ is a total constraint side with end points $A_{j}, A_{j+1}$ and $u \in \mathscr{H} \mathscr{P}_{p}(\Omega)$, then $u_{i}\left(A_{j}\right)=u_{i}\left(A_{j+1}\right)=0, i=1,2$, and

$$
\int_{\Gamma_{j}} u_{i} \psi_{i} d s=0 \quad \text { for all } \psi_{i} \in{ }^{1} \mathscr{P}_{p-2}\left(\Gamma_{j}\right), i=1,2 \text {. }
$$

(ii) If $\Gamma_{j}$ is a partial constraint side, then

$$
\left(\alpha^{(j)} u_{1}+\beta^{(j)} u_{2}\right)\left(A_{k}\right)=0, \quad k=j, j+1,
$$

and

$$
\int_{\Gamma_{j}}\left(\alpha^{(j)} u_{1}+\beta^{(j)} u_{2}\right) \psi d s=0 \quad \text { for all } \psi \in \epsilon^{2} \mathscr{P}_{p-2}\left(\Gamma_{j}\right) .
$$

The $\rho$-hyperplane ${ }_{\rho}^{\mathscr{H}} \mathscr{P}_{p}(\Omega)$ is defined analogously. Let $g$ be defined in terms of $\rho$ by (2.8). Then on $\Gamma_{j}$ we impose

$$
u_{i}\left(A_{k}\right)=g_{i}^{(j)}\left(A_{k}\right), \quad i=1,2, k=j, j+1,
$$

respectively

$$
\left(\alpha^{(j)} u_{1}+\beta^{(j)} u_{2}\right)\left(A_{k}\right)=g^{(j)}\left(A_{k}\right), \quad k=j, j+1,
$$

and

$$
\int_{\Gamma_{j}} u_{i} \psi_{i} d s=\int_{\Gamma_{j}} g_{i}^{(j)} \psi_{i} d s, \quad i=1,2
$$

respectively

$$
\int_{\Gamma_{j}}\left(\alpha^{(j)} u_{1}+\beta^{(j)} u_{2}\right) \psi d s=\int_{\Gamma_{j}} g^{(j)} \psi d s .
$$

The $p$-version is then defined analogously as before: Find $u_{p} \in \mathscr{H}_{\rho}^{\mathscr{P}} \mathscr{P}_{p}(\Omega)$ such that

$$
B\left(u_{p}, v\right)=G_{1}(v) \quad \forall v \in \mathscr{\mathscr { H }}_{p}(\Omega) .
$$

Remark. Constraints of the type considered are typical in elasticity theory. Here, $u_{1}$ and $u_{2}$ are the displacements in the directions $x_{1}$ and $x_{2}$, respectively. Assume now that the displacement is constrained in the normal direction only (and is friction-free in the tangential direction). Then on the boundary, we obtain the partial constraint $u_{1} \cos \varphi+u_{2} \sin \varphi=0$, where $\varphi$ is the angle of the outer normal with the axis $x_{1}$. 


\section{The Convergence of the $p$-Version of the Finite Element Method.}

3.1. An Abstract Result. We will first describe an abstract framework which will be the basis for the forthcoming analysis.

Let $X$ and $W$ be Hilbert spaces and $\tilde{X}_{p} \subset X, \tilde{W}_{p} \subset W, p=1,2, \ldots$, be oneparameter families of finite-dimensional subspaces. $X_{p} \subset X, W_{p} \subset W$ will denote corresponding families of hyperplanes such that $(u-v) \in \tilde{X}_{p}$ whenever $u, v \in X_{p}$, and $(\varphi-\psi) \in \tilde{W}_{p}$ whenever $\varphi, \psi \in W_{p}$.

Let $a(u, v), u, v \in X$, be a continuous bilinear form on $X \times X$ and $b(v, \varphi)$ be a continuous bilinear form on $X \times W$ such that $b(v, \varphi) \leq C\|v\|_{X}\|\varphi\|_{W}$.

Let $u_{0} \in X, \varphi_{0} \in W$ and $u_{p} \in X_{p}, \varphi_{p} \in W_{p}$ be such that

$$
\begin{gathered}
a\left(u_{0}, v\right)+b\left(v, \varphi_{0}\right)=F_{1}(v) \quad \forall v \in \tilde{X}_{p} \\
b\left(u_{0}, \psi\right)=F_{2}(\psi) \quad \forall \psi \in \tilde{W}_{p}
\end{gathered}
$$

and

$$
\begin{gathered}
a\left(u_{p}, v\right)+b\left(v, \varphi_{p}\right)=F_{1}(v) \quad \forall v \in \tilde{X}_{p} \\
b\left(u_{p}, \psi\right)=F_{2}(\psi) \quad \forall \psi \in \tilde{W}_{p} .
\end{gathered}
$$

Define $Z_{p}=\left\{v \in \tilde{X}_{p}, b(v, \psi)=0 \forall \psi \in \tilde{W}_{p}\right\} \subset \tilde{X}_{p}$. Then we have

THEOREM 3.1. Let $a(u, u) \geq \gamma\|u\|_{X}^{2}, \gamma>0$, for any $u \in Z_{p}$. Then

$$
\left\|u_{0}-u_{p}\right\|_{X} \leq C\left[\inf _{\substack{b\left(u_{0}-w_{p}, \psi\right)=0 \\ u_{p}-w_{p} \in \tilde{X}_{p} \\\left(i . e ., u_{p}-w_{p} \in Z_{p}\right)}}\left\|u_{0}-w_{p}\right\|_{X}+\inf _{\chi_{p} \in W_{p}}\left\|\varphi_{0}-\chi_{p}\right\|_{W}\right] .
$$

Proof. For arbitrary $w_{p} \in X_{p}$ and $\chi_{p} \in W_{p}$ we have

$$
\begin{aligned}
& a\left(u_{p}-w_{p}, v\right)+b\left(v, \varphi_{p}-\chi_{p}\right) \\
& \quad=a\left(u_{0}-w_{p}, v\right)+b\left(v, \varphi_{0}-\chi_{p}\right) \quad \forall v \in \widetilde{X}_{p} \\
& b\left(u_{p}-w_{p}, \psi\right)=b\left(u_{0}-w_{p}, \psi\right) \quad \forall \psi \in \widetilde{W}_{p} .
\end{aligned}
$$

For $v \in Z_{p},(3.4 \mathrm{a})$ yields

$$
a\left(u_{p}-w_{p}, v\right)=a\left(u_{0}-w_{p}, v\right)+b\left(v, \varphi_{0}-\chi_{p}\right) .
$$

Suppose now that $w_{p}$ is such that

$$
b\left(w_{p}, \psi\right)=b\left(u_{0}, \psi\right) \quad \forall \psi \in \widetilde{W}_{p}
$$

Then, by $(3.4 b)$,

$$
b\left(u_{p}-w_{p}, \psi\right)=0 \quad \forall \psi \in \widetilde{W}_{p}
$$

and hence

$$
u_{p}-w_{p} \in Z_{p}
$$

Now using $v=u_{p}-w_{p}$ in (3.5), we get

$$
a\left(u_{p}-w_{p}, u_{p}-w_{p}\right) \leq C\left[\left\|u_{0}-w_{p}\right\|_{X}\left\|u_{p}-w_{p}\right\|_{X}+\left\|\varphi_{0}-\chi_{p}\right\|_{W}\left\|u_{p}-w_{p}\right\|_{X}\right]
$$


and hence, by coercivity of $a(\cdot, \cdot)$ on $Z_{p}$,

$$
\left\|u_{p}-w_{p}\right\|_{X} \leq C\left[\left\|u_{0}-w_{p}\right\|_{X}+\left\|\varphi_{0}-\chi_{p}\right\|_{W}\right]
$$

and hence also

$$
\left\|u_{0}-w_{p}\right\|_{X} \leq C\left[\left\|u_{0}-w_{p}\right\|_{X}+\left\|\varphi_{0}-\chi_{p}\right\|_{W}\right],
$$

from which (3.3) follows.

3.2. The Convergence of the $p$-Version. Let $u_{0}=\left(u_{0,1}, u_{0,2}\right) \in{ }^{2} H^{1}(\Omega)$ be the solution of our constrained problem $(2.2),(2.5)$ and $u_{p}=\left(u_{p, 1}, u_{p, 2}\right) \in{ }^{2} \mathscr{P}_{p}(\Omega)$ be the approximation given by $(2.10)$.

We will assume that $u_{0} \in{ }^{2} H^{k}(\Omega), k>3 / 2$. Hence $\partial u_{0} / \partial n \in{ }^{2} H^{0}\left(\Gamma_{i}\right)$, $i=1,2, \ldots, m$. Let the constraints on $\Gamma_{j}$ be as in Subsection 2.2, with $\Gamma_{i_{j}}$, $j=1, \ldots, m_{1}$, being the total constraint sides and $\Gamma_{i_{j}}, j=m_{1}+1, \ldots, m$, the partial constraint sides. Then it can be verified that for any $v \in{ }^{2} H^{1}(\Omega)$,

$$
\begin{aligned}
B\left(u_{0}, v\right) & -\sum_{j=1}^{m_{1}} \int_{\Gamma_{i_{j}}}\left(\frac{\partial u_{0,1}}{\partial n} v_{1}+\frac{\partial u_{0,2}}{\partial n} v_{2}\right) d s \\
& -\sum_{j=m+1}^{m} \int_{\Gamma_{i_{j}}}\left(\alpha^{i_{j}} v_{1}+\beta^{i_{j}} v_{2}\right)\left(\frac{\partial u_{0,1}}{\partial n}+\frac{\partial u_{0,2}}{\partial n}\right) d s=G_{1}(v),
\end{aligned}
$$

where we have assumed $\alpha^{i_{j}}+\beta^{i_{j}}=1$. Moreover, for $\psi, \psi_{1}, \psi_{2}$ and $\rho$ as in (2.8)$(2.9)$,

$$
\begin{aligned}
& \sum_{j=1}^{m_{1}} \int_{\Gamma_{i_{j}}}\left(u_{p, 1} \psi_{1}+u_{p, 2} \psi_{2}\right) d s+\sum_{j=m_{1}+1}^{m} \int_{\Gamma_{i_{j}}}\left(\alpha^{i_{j}} u_{p, 1}+\beta^{i_{j}} u_{p, 2}\right) \psi d s \\
& \quad=\sum_{j=1}^{m_{1}} \int_{\Gamma_{i_{j}}}\left(u_{0,1} \psi_{1}+u_{0,2} \psi_{2}\right) d s+\sum_{j=m_{1}+1}^{m} \int_{\Gamma_{i_{j}}}\left(\alpha^{i_{j}} u_{0,1}+\beta^{i_{j}} u_{0,2}\right) \psi d s \\
& \quad=\sum_{j=1}^{m_{1}} \int_{\Gamma_{i_{j}}}\left(\rho_{1} \psi_{1}+\rho_{2} \psi_{2}\right) d s+\sum_{j=m_{1}+1}^{m} \int_{\Gamma_{i_{j}}}\left(\alpha^{i_{j}} \rho_{1}+\beta^{i_{j}} \rho_{2}\right) \psi d s
\end{aligned}
$$

and

$$
\begin{array}{cl}
u_{p, k}\left(A_{j}\right)=u_{0, k}\left(A_{j}\right)=\rho_{k}\left(A_{j}\right), \quad j=l, l+1, k=1,2, & \\
& l=i_{1}, \ldots, i_{m_{1}}, \\
\left(\alpha^{l} u_{p, 1}\right)\left(A_{j}\right)+\left(\beta^{l} u_{p, 2}\right)\left(A_{j}\right)=\left(\alpha^{l} u_{0,1}\right)\left(A_{j}\right)+\left(\beta^{l} u_{0,2}\right)\left(A_{j}\right) \\
=\left(\alpha^{l} \rho_{1}\right)\left(A_{j}\right)+\left(\beta^{l} \rho_{2}\right)\left(A_{j}\right), \quad j=l, l+1, l=i_{m_{1}+1}, \ldots, i_{m} .
\end{array}
$$

(We remark that $u_{0, i}\left(A_{j}\right), i=1,2$, has meaning because we assumed that $u_{0} \in$ ${ }^{2} H^{k}(\Omega), k>3 / 2$.)

We now define

$$
X={ }^{2} H^{1}(\Omega), \quad\|\cdot\|_{X}=\|\cdot\|_{2} H^{1}(\Omega)
$$

and for any $\delta=\left(\delta_{1}, \delta_{2}\right) \in X$,

$$
\begin{aligned}
X_{p, \delta}=\{u= & \left(u_{1}, u_{2}\right) \in{ }^{2} \mathscr{P}_{p}(\Omega), u_{k}\left(A_{j}\right)=\delta_{k}\left(A_{j}\right), k=1,2, \\
& j=l, l+1, l=i_{1}, \ldots, i_{m_{1}},\left(\alpha^{l} u_{1}\right)\left(A_{j}\right)+\left(\beta^{l} u_{2}\right)\left(A_{j}\right) \\
= & \left.\left(\alpha^{l} \delta_{1}\right)\left(A_{j}\right)+\left(\beta^{l} \delta_{2}\right)\left(A_{j}\right), j=l, l+1, l=i_{m_{1}+1}, \ldots, i_{m}\right\} .
\end{aligned}
$$


We then take in our abstract framework

$$
X_{p}=X_{p, \rho}, \quad \tilde{X}_{p}=X_{p, 0},
$$

where $\rho$ satisfies (2.6)-(2.8). Moreover, let

$$
W=\prod_{j=1}^{m_{1}}{ }^{2} H^{-1 / 2}\left(\Gamma_{i_{j}}\right) \times \prod_{j=m_{1}+1}^{m}{ }^{1} H^{-1 / 2}\left(\Gamma_{i_{j}}\right)
$$

with the norm

$$
\|\psi\|_{W}=\left[\sum_{j=1}^{m_{1}}\left\|\psi_{j}\right\|_{{ }^{-1 / 2}\left(\Gamma_{i_{j}}\right)}^{2}+\sum_{j=m_{1}+1}^{m}\left\|\psi_{j}\right\|_{{ }^{-1 / 2}\left(\Gamma_{i_{j}}\right)}^{2}\right]^{1 / 2} .
$$

We then see that $\varphi_{0} \in W$, where (see (3.6))

$$
\begin{aligned}
\varphi_{0, j} & =-\left(\left.\frac{\partial u_{0,1}}{\partial n}\right|_{\Gamma_{i_{j}}},\left.\frac{\partial u_{0,2}}{\partial n}\right|_{\Gamma_{i_{j}}}\right), \quad j=1, \ldots, m_{1} \\
& =-\left.\left(\frac{\partial u_{0,1}}{\partial n}+\frac{\partial u_{0,2}}{\partial n}\right)\right|_{\Gamma_{i_{j}}}, \quad j=m_{1}+1, \ldots, m .
\end{aligned}
$$

Define $W_{p}=\widetilde{W}_{p}=\prod_{j-1}^{m}{ }_{j} W_{p} \subset W$, where

$$
\begin{aligned}
{ }_{j} W_{p} & ={ }^{2} H^{-1 / 2}\left(\Gamma_{i_{j}}\right) \cap{ }^{2} \mathscr{P}_{p-2}\left(\Gamma_{j}\right), & & j=1, \ldots, m_{1} \\
& ={ }^{1} H^{-1 / 2}\left(\Gamma_{i_{j}}\right) \cap{ }^{1} \mathscr{P}_{p-2}\left(\Gamma_{j}\right), & & j=m_{1}+1, \ldots, m .
\end{aligned}
$$

Let $a(\cdot, \cdot)$ and $b(\cdot, \cdot)$ be bilinear forms defined respectively on $X \times X$ and $X \times W$ by

$$
\begin{gathered}
a(u, v)=B(u, v), \\
b(u, \psi)=\sum_{j=1}^{m_{1}} \int_{\Gamma_{i_{j}}}\left(u_{1} \psi_{1}+u_{2} \psi_{2}\right) d s+\sum_{j=m_{1}+1}^{m} \int_{\Gamma_{i_{j}}}\left(\alpha^{i_{j}} u_{1}+\beta^{i_{j}} u_{2}\right) \psi d s .
\end{gathered}
$$

It may be seen that the right-hand side of (3.7) defines a linear functional $G_{2}$ on $W_{p}$. Then (3.6)-(3.8) show that $\left(u_{0}, \varphi_{0}\right)$ satisfy (3.1) with $F_{k}=G_{k}, k=1,2$. Moreover, if we can find a unique pair $\left(u_{p}, \varphi_{p}\right)$ satisfying $(3.2)$, then $u_{p}$ will be precisely our finite element solution satisfying (2.10). We will now verify that the mixed method defined above satisfies the assumptions of Theorem 3.1. This in turn will lead to the existence and uniqueness of the solution $\left(u_{p}, \varphi_{p}\right)$ of $(3.2)$ and an estimate of the rate of convergence of $u_{p}$ to $u$.

Obviously, $a(u, v)$ satisfies the desired continuity and coercivity conditions. For $j=1,2, \ldots, m$ we have

$$
\left|\int_{\Gamma_{i_{j}}} u \psi d s\right| \leq C\|u\|_{H^{1 / 2}\left(\Gamma_{i_{j}}\right)}\|\psi\|_{H^{-1 / 2}\left(\Gamma_{i_{j}}\right)}
$$

from which the continuity of $b(\cdot, \cdot)$ may be deduced. Hence Theorem 3.1 is applicable. Let us now estimate $\inf _{\chi \in W_{p}}\left\|\varphi_{0}-\chi\right\|_{W}$. First, let $m_{1}+1 \leq j \leq m$. We assumed that $\varphi_{0, j} \in H^{k-3 / 2}\left(\Gamma_{i_{j}}\right), k>3 / 2$. Hence, $\varphi_{0, j}\left(F_{i_{j}}(\xi)\right)=\varphi(\xi) \in H^{k-3 / 2}(I)$. Let $\sigma \in{ }^{1} \mathscr{P}_{p-2}(I)$ be such that

$$
\int_{I} \sigma \rho d \xi=\int_{I} \varphi \rho d \xi \quad \forall \rho \in{ }^{1} \mathscr{P}_{p-2}(I) .
$$


Then, with $q=\varphi-\sigma$, we have

$$
\|q\|_{H^{0}(I)} \leq C p^{-(k-3 / 2)}\|\varphi\|_{H^{k-3 / 2}(I)} .
$$

Now, for arbitrary $v \in H^{1}(I)$, we have by (3.9)

$$
\frac{\int_{I} q v d \xi}{\|v\|_{H^{1}(I)}}=\frac{\int_{I} q\left(v-\sigma_{1}\right) d \xi}{\|v\|_{H^{1}(I)}} \leq \frac{\|q\|_{H^{0}(I)}\left\|v-\sigma_{1}\right\|_{H^{0}(I)}}{\|v\|_{H^{1}(I)}} \leq C p^{-1}\|q\|_{H^{0}(I)}
$$

where $\sigma_{1}$ is a polynomial of degree $p-2$ satisfying

$$
\left\|v-\sigma_{1}\right\|_{H^{0}(I)} \leq C p^{-1}\|v\|_{H^{1}(I)} .
$$

This yields

$$
\|q\|_{H^{-1}(I)} \leq C p^{-(k-1 / 2)}\|\varphi\|_{H^{k-3 / 2}(I)} .
$$

Interpolating (3.10), (3.11) and using the fact that $F_{i_{j}}$ is a smooth mapping, we obtain

$$
\inf _{\chi_{p} \in W_{j}}\left\|\varphi_{0, j}-\chi_{p}\right\|_{H^{-1 / 2}\left(\Gamma_{i_{j}}\right)} \leq C p^{-(k-1)}\left\|\varphi_{0, j}\right\|_{H^{k-3 / 2}\left(\Gamma_{i_{j}}\right)} .
$$

We get similar estimates for $\Gamma_{i_{j}}, j=1, \ldots, m_{1}$, so that

$$
\inf _{\chi_{p} \in W_{p}}\left\|\varphi_{0}-\chi_{p}\right\|_{W} \leq C p^{-(k-1)}\left\|u_{0}\right\|_{2} H^{k}(\Omega) .
$$

We now estimate inf $\left\|u_{0}-w_{p}\right\|_{X}$. Using the results from [2], there exist $z_{i} \in{ }^{1} \mathscr{P}_{p}(\Omega)$, $i=1,2$, such that

$$
\begin{aligned}
& \left\|u_{0, i}-z_{i}\right\|_{H^{t}(\Omega)} \leq C p^{-(k-t)}\left\|u_{0, i}\right\|_{H^{k}(\Omega)}, \quad i=1,2, t=0,1, \\
& u_{0, i}(N)=z_{i}(N) \quad \text { for each node } N \text { of the mesh, } \\
& \left\|u_{0, i}-z_{i}\right\|_{H^{t}\left(\Gamma_{j}\right)} \leq C p^{-(k-1 / 2-t)}\left\|u_{0, i}\right\|_{H^{k}(\Omega)}, \quad t=0,1, i=1,2, \\
& j=1,2, \ldots, m .
\end{aligned}
$$

Let $m_{1}+1 \leq j \leq m$. Let us denote $\kappa=\alpha^{i_{j}}\left(u_{0,1}-z_{1}\right)+\beta^{i_{j}}\left(u_{0,2}-z_{2}\right)$. Then we have $\kappa\left(A_{i_{j}}\right)=\kappa\left(A_{i_{j}+1}\right)=0$. Let $\tilde{\kappa}(\xi)=\kappa(F(\xi))$ and let $\tau(F(\xi))=\tilde{\tau}(\xi) \in{ }^{1} \mathscr{P}_{p}(I)$ satisfy $\tilde{\tau}( \pm 1)=0$ and

$$
\int_{I} \tilde{\tau} v d \xi=\int_{I} \tilde{\kappa} v d \xi \quad \forall v \in{ }^{1} \mathscr{P}_{p-2}(I) .
$$

Because of $(3.13 \mathrm{~b})$ we can write

$$
\int_{I} \tilde{\tau}^{\prime} \omega^{\prime} d \xi=\int_{I} \tilde{\kappa}^{\prime} \omega^{\prime} d \xi \quad \forall \omega \in{ }^{1} \mathscr{P}_{p}(I), \omega( \pm 1)=0
$$

and hence by Lemma 3.2 of [2]

$$
\|\tilde{\kappa}-\tilde{\tau}\|_{H^{t}(I)} \leq C p^{-(k-1 / 2-t)}\left\|u_{0}\right\|_{{ }^{2} H^{k}(\Omega)}, \quad t=0,1 .
$$

Using (3.13c), this gives

$$
\|\tilde{\tau}\|_{H^{t}(I)} \leq C p^{-(k-1 / 2-t)}\left\|u_{0}\right\|_{{ }^{2} H^{k}(\Omega)}, \quad t=0,1 .
$$

Now using Lemma 2.1 , it follows that there is a $w \in{ }^{1} \mathscr{P}_{p}(\Omega)$ such that $w=0$ on $\Omega-\tilde{\Omega}$, where $\tilde{\Omega}$ is the element with the side $\Gamma_{i_{j}}, w=\tau$ on $\Gamma_{i_{j}}, w=0$ on $\partial \tilde{\Omega}-\Gamma_{i_{j}}$ and

$$
\|w\|_{H^{1}(\Omega)} \leq C p^{-(k-1)}\left\|u_{0}\right\|_{2 H^{k}(\Omega)} .
$$


Letting $w_{i_{j}}=(w, w) \in{ }^{2} H^{1}(\Omega)$, we see that $w_{i_{j}}$ will satisfy $(3.14)$ with $H^{1}(\Omega)$ replaced by ${ }^{2} H^{1}(\Omega)$.

Let now $w_{p}=z+w_{i_{j}}=\left(z_{1}, z_{2}\right)+(w, w)$. Using $(3.13 \mathrm{~b})$ and the fact that $w\left(A_{l}\right)=0$, we obtain

$$
\left(\alpha^{i_{j}}\left(u_{0,1}-w_{p, 1}\right)+\beta^{i_{j}}\left(u_{0,2}-w_{p, 2}\right)\right)\left(A_{l}\right)=0, \quad l=i_{j}, i_{j}+1 .
$$

Moreover, for $\psi \in{ }^{1} \mathscr{P}_{p-2}\left(\Gamma_{i_{j}}\right)$,

$$
\begin{aligned}
& \int_{\Gamma_{i_{j}}}\left(\alpha^{i_{j}}\left(u_{0,1}-w_{p, 1}\right)+\beta^{i_{j}}\left(u_{0,2}-w_{p, 2}\right)\right) \psi d s \\
& \quad=\int_{\Gamma_{i_{j}}}\left(\kappa-\left(\alpha^{i_{j}}+\beta^{i_{j}}\right) w\right) \psi d s=\int_{\Gamma_{i_{j}}}(\kappa-w) \psi d s=0,
\end{aligned}
$$

where we have used $\alpha^{i_{j}}+\beta^{i_{j}}=1$. We may construct $w_{i_{j}}$ as above for all partial constraint sides. An analogous construction can be carried out for total constraint sides as well. Then, if

$$
w_{p}=z+\sum_{j=1}^{m} w_{i_{j}}
$$

we see that

$$
\begin{gathered}
u_{p}-w_{p} \in \tilde{X}_{p} \\
b\left(u_{0}-w_{p}, \psi\right)=0 \quad \forall \psi \in W_{p}
\end{gathered}
$$

and

$$
\left\|u_{0}-w_{p}\right\|_{X} \leq\left\|u_{0}-z\right\|_{X}+\sum_{j=1}^{m}\left\|w_{i_{j}}\right\|_{X} \leq C p^{-(k-1)}\left\|u_{0}\right\|_{2} H^{k}(\Omega) .
$$

This provides a bound for the first term in the right-hand side of (3.3). Hence we have proven

THEOREM 3.2. Let $u_{0} \in{ }^{2} H^{k}(\Omega), k>3 / 2$. Then

$$
\left\|u_{0}-u_{p}\right\|_{2 H^{1}(\Omega)} \leq C p^{-(k-1)}\left\|u_{0}\right\|_{2 H^{k}(\Omega)},
$$

where $u_{0}$ is the exact solution and $u_{p}$ is the finite element solution of the constrained problem, provided that $u_{0}$ and $u_{p}$ exist.

The next theorem deals with the question of existence and uniqueness of $\left(u_{0}, \varphi_{0}\right)$ and $\left(u_{p}, \varphi_{p}\right)$.

THEOREM 3.3. The (exact) solution $\left(u_{0}, \varphi_{0}\right)$ of the constrained problem exists. The finite element solution $\left(u_{p}, \varphi_{p}\right)$ exists and is unique.

Proof. In Subsection 2.2 we have shown that $u_{0}$ exists and hence $\left(u_{0}, \varphi_{0}\right)$ exists, too. The finite element solution $\left(u_{p}, \varphi_{p}\right)$ is determined by the solution of a linear system of equations with square matrix. Hence the existence follows from the uniqueness. Assume therefore that there is a solution $\left(u_{p}, \varphi_{p}\right)$ of the trivial problem. Obviously $\bar{u}=\bar{\varphi}=0$ is also a solution of this problem. Hence $u_{p}=0$ because of Theorem 3.1. We have to show, therefore, that

$$
\int_{I}\left(\alpha v_{1}+\beta v_{2}\right) \varphi_{p} d \xi=0
$$


implies $\varphi_{p}=0$. Because $\alpha+\beta=1$ we also have $\int_{I} v \varphi_{p} d \xi=0$ for all $v \in$ ${ }^{1} \mathscr{P}_{p}(I) \cap H_{0}^{1}(I)$ while $\varphi_{p} \in{ }^{1} \mathscr{P}_{p-2}(I)$. This leads to $\varphi_{p}=0$, which leads to the desired result.

Remark. We have dealt only with a model problem. It is obvious that the theorem holds in general, as for example for the theory of elasticity.

4. Some Aspects of Implementation. Here we will make some comments about the implementation in the framework of the code $\mathrm{PROBE}^{* * *}$ (see [6]). The shape functions are defined as usual on the standard square or triangle. There are three types:

(a) the nodal shape functions which are linear on every side of $Q$, respectively $T$;

(b) the side functions which are zero at the vertices of $Q$, respectively $T$, and on $\gamma$ are of the form

$$
\xi_{j}=\int_{-1}^{X} l_{j}(\xi) d \xi, \quad j=1,2, \ldots,
$$

where $l_{j}$ is the Legendre polynomial of degree $j . \xi_{j}$ is then a polynomial of degree $j+1$

(c) The internal shape functions which are zero on $\partial Q$ (respectively $\partial T$ ).

The stiffness matrices are first computed in the standard way without constraints. Then the constraints are imposed at the vertices $A_{j}$. This only involves the amplitudes for the nodal shape functions. Then the conditions $(2.9 \mathrm{a}, \mathrm{b})$ only involve amplitudes for the side shape functions. The functions $\psi$ in $(2.9 \mathrm{a}, \mathrm{b})$ are computed as derivatives of the Legendre polynomials from the usual recurrence formula and the integration is made using numerical quadrature.

The condition (2.9a) is especially simple because $u_{i}=\sum c_{i} \xi_{i}$. Integrating by parts and exploiting orthogonality of the Legendre polynomials, we get the amplitudes for the side shape functions on the total constraint sides directly.

Institute for Physical Science and Technology

University of Maryland

College Park, Maryland 20742

Department of Mathematics

University of Maryland Baltimore County

Catonsville, Maryland 21228

1. I. BABUSKKa, The $p$ and h-p Versions of the Finite Element Method, The State of the Art, Technical Note BN-1156, Institute for Physical Science and Technology, University of Maryland, 1986.

2. I. BABUŠKA \& M. SURI, "The optimal convergence rate of the p-version of the finite element method," SIAM J. Numer. Anal., v. 24, 1987, pp. 750-776.

3. I. BABUSKKA \& M. SURI, "The $h-p$ version of the finite element method with quasiuniform meshes," RAIRO Modél. Math. Anal. Numér., v. 21, 1987, pp. 199-238.

4. I. BABUSKKA \& M. SURI, The Treatment of Nonhomogeneous Dirichlet Boundary Conditions by the $p$-Version of the Finite Element Method, Technical Note BN-1063, Institute for Physical Science and Technology, University of Maryland, 1987.

5. J. L. LiONS \& E. MAGEnES, Non-Homogeneous Boundary Value Problems and Applications-I, Springer-Verlag, New York, Heidelberg, Berlin, 1972.

6. B. A. SZABÓ, PROBE: Theoretical Manual, Noetic Technologies Corporation, St. Louis, Missouri, 1985.

*** The code PROBE is the code of Noetic Tech., St Louis. 